\title{
Inhaltsverzeichnis
}

\author{
Abkürzungsverzeichnis - IX
}

Abbildungsverzeichnis $-\mathrm{XI}$

Tabellenverzeichnis - XIII

Kurzfassung $-\mathrm{XIX}$

Abstract $\longrightarrow \mathbf{X X}$

1 Einleitung - 1

1.1 Ausgangslage -1

1.2 Stand der Forschung -6

1.3 Forschungslücke -13

1.4 Zielsetzung -14

1.5 Struktur der Arbeit -14

\section{Methodik 16}

2.1 Literaturrecherche -21

2.2 Experteninterviews -21

2.3 Prototyp Berechnungswerkzeug $\mathbf{2 6}$

2.4 Verifizierung und Validierung 26

2.5 Plausibilisierung 32

2.6 Praxisbeispiel $\mathbf{3 5}$

2.7 Dokumentation -36

3 Grundlagen Laborgebäude - 38

3.1 Definition Laborgebäude - 38

3.2 Typologie Laborgebäude $-\mathbf{3 9}$

3.2.1 Parameter und planerische Tendenzen - 39

3.2.2 Nutzungsbereiche und Raumprogramm — 41

3.2.3 Baukonstruktion -43

3.2.4 Technische Anlagen -45

3.3 Eingrenzung Untersuchungsgegenstand -47 


\section{Grundlagen Lebenszykluskosten -53}

4.1 Lebenszyklus einer Immobilie - $\mathbf{5 3}$

4.2 Lebenszykluskosten und -strukturen - 53

4.2.1 Lebenszykluskosten $\mathbf{5 4}$

4.2.2 Lebenszykluserfolg -55

4.2.3 Lebenszykluskostenstrukturen $-\mathbf{5 6}$

4.2.3.1 Herstellungskosten $-\mathbf{5 8}$

4.2.3.2 Nutzungskosten -59

4.2.3.3 Mengen und Flächen -60

4.2.4 Abgrenzung und Definition Instandsetzung - 65

4.3 Lebenszykluskostenermittlung $\mathbf{7 0}$

4.3.1 Kapitalwertmethode bzw. Barwertmethode - 73

4.4 Eingangsgrößen Lebenszykluskostenermittlung 75

4.4.1 Gebäudeart und Standortbedingungen - 75

4.4.2 Systemgrenze -75

4.4.3 Art und Umfang der Kosten -76

4.4.4 Detaillierungsgrad der Kostenermittlung — 76

4.4.5 Bezugsgröße der Kosten -77

4.4.6 Mehrwertsteuer in Deutschland und der Schweiz - 78

4.4.7 Betrachtungszeitraum $\mathbf{7 8}$

4.4.8 Kalkulations- bzw. Diskontierungszinsssatz 79

4.4.9 Preisänderung -82

4.4.10 Kennwerte $\mathbf{8 3}$

4.4.11 Preise und Stundenverrechnungssätze -83

4.4.12 Preisstand der Bau- und Nutzungskosten - $\mathbf{8 3}$

4.4.13 Regionalfaktoren -83

4.4.14 Nutzungsdauer von Bauteilen -83

4.5 Zusammenfassung $\mathbf{8 4}$

\section{Entwicklung Berechnungswerkzeug - 85}

5.1 Struktur des Berechnungswerkszeugs -85

\subsubsection{Software -85}

5.1.2 Grafische Gestaltung - 86

5.1.3 Deutsche und schweizerische Version - $\mathbf{8 7}$

5.1.4 Aufbau des Berechnungswerkzeugs $\mathbf{8 7}$

5.1.5 Eingabe ins Berechnungswerkzeug $\mathbf{8 8}$

5.1.6 Systemgrenze $\mathbf{8 8}$

5.1.7 Umfang der Kosten - $\mathbf{8 9}$

5.1.8 Detaillierungsgrad der Kostenermittlung - 89

5.1.9 Bezugsgröße der Kosten mit Mehrwertsteuer $\mathbf{8 9}$

5.1.10 Berechnungsparameter $\mathbf{9 0}$

5.1.11 Risikozuschläge $-\mathbf{9 0}$ 
5.1.12 Preisstand der Bau- und Nutzungskosten $-\mathbf{9 0}$

5.1.13 Kennwerte- und Kennzahlen - 90

5.1.14 Tarife und Stundenverrechnungssätze - 91

5.2 Kosteneinflussfaktoren auf Nutzungskosten von Laborgebäuden — 91

5.2.1 Objektmanagementkosten -93

5.2.2 Versorgungskosten -94

5.2.3 Entsorgungskosten -95

5.2.4 Kosten für Reinigung und Pflege von Gebäuden -95

5.2.5 Kosten für Bedienung, Inspektion und Wartung - 95

5.2.6 Kosten für Sicherheits- und Überwachungsdienste -96

5.2.7 Abgaben und Beiträge - 96

5.2.8 Instandsetzung der Baukonstruktion - 96

5.2.9 Instandsetzung der technischen Anlagen $\mathbf{1 0 0}$

5.3 Objektmanagementkosten Forschungs- und Laborgebäude 103

5.3.1 KG 200 Objektmanagement 103

5.3.2 Faktor nach Optimierung -104

5.3.3 Faktoren nach Fall-Kontroll-Studie 104

5.4 Betriebskosten Forschungs- und Laborgebäude 105

5.4.1 KG 310 Versorgung 105

5.4.1.1 Faktoren nach Optimierung 106

5.4.1.2 Faktoren nach Fall-Kontroll-Studie - 106

5.4.2 KG 320 Entsorgung 109

5.4.2.1 Faktoren nach Optimierung $\mathbf{1 1 0}$

5.4.2.2 Faktoren nach Fall-Kontroll-Studie - 111

5.4.3 KG 330 Reinigung und Pflege von Gebäuden - 112

5.4.3.1 Faktoren nach Optimierung — 113

5.4.3.2 Faktoren nach Fall-Kontroll-Studie - 113

5.4.4 KG 350 Bedienung Inspektion und Wartung — 116

5.4.4.1 Faktoren nach Optimierung - 117

5.4.4.2 Faktoren nach Fall-Kontroll-Studie - 118

5.4.5 KG 360 Sicherheits- und Überwachungsdienste 125

5.4.5.1 Faktor nach Optimierung - 125

5.4.5.2 Faktoren nach Fall-Kontroll-Studie 126

5.4.6 KG 370 Abgaben und Beiträge 126

5.4.7 Faktor nach Optimierung 126

5.4.8 Faktoren nach Fall-Kontroll-Studie 126

5.5 Instandsetzungskosten Forschungs- und Laborgebäude 127

5.5.1 KG 410 Instandsetzung der Baukonstruktionen - 127

5.5.2 KG 420 Instandsetzung der Technischen Anlagen 129

5.6 Zusammenfassung 131 
VIII Inhaltsverzeichnis

6 Anwendung Berechnungswerkzeug - 133

6.1 Eingangswerte und Szenarien -133

6.2 Optimierung der Nutzungskosten -144

7 Fazit und Ausblick -147

Literaturverzeichnis -159

Anhang A: Experteninterviews - Vorabinformation - 169

Anhang B: Experteninterviews - Befragungsbogen -171

Anhang C: Einflussfaktoren auf Nutzungskosten und Nutzungsdauern

Anhang D: Erhebungsbogen 271

Anhang E: Eingangsgrössen Lebenszykluskostenermittlung - 313

Danksagung - 343 